\title{
Compound gastroenteropancreatic neuroendocrine and gastrointestinal stromal tumors in the stomach: A case report
}

\author{
YU ZHOU ${ }^{1}$, PING CHEN $^{1}$ and LIANG ZHONG ${ }^{1,2}$ \\ ${ }^{1}$ Department of Gastrointestinal Surgery, Clinical Medical College of Yangzhou University, Subei People's Hospital of Jiangsu, \\ Yangzhou, Jiangsu 225001, P.R. China; ${ }^{2}$ Department of Gastrointestinal Surgery, Graduate School of Medicine, \\ University of Tokyo, Tokyo 113-0033, Japan
}

Received November 6, 2014; Accepted July 7, 2015

DOI: $10.3892 / 01.2015 .3666$

\begin{abstract}
Gastroenteropancreatic neuroendocrine tumors (GEP-NETs) combined with gastrointestinal stromal tumors (GISTs) are rarely observed in the clinic. In the present study, the case of a 56-year-old female diagnosed with compound GEP-NETs and GISTs was reported. The patient initially presented with epigastric discomfort. The pre-operative diagnosis was of GISTs based on the endoscopic and imaging findings. A subtotal gastrectomy and Roux-en-Y reconstruction were successfully performed. The final diagnosis was revised to be compound GEP-NETs and GISTs based on the pathological findings. After 17 months of follow-up examinations using computed tomography and ultrasonography, the patient showed no symptoms or signs of recurrence.
\end{abstract}

\section{Introduction}

Gastroenteropancreatic neuroendocrine tumors (GEP-NETs) are extremely rare tumors ( $<1 \%$ of stomach tumors) that usually originate from the neuroendocrine tissues of the digestive system. Driver mutations in MEN1, DAXX or ATRX and mTOR pathway genes have been identified as crucial factors in GEP-NETs tumorigenesis (1). The first case of GEP-NETs was reported approximately one century ago. Recently, the incidence of these tumors has greatly increased $(2,3)$. According to the Epidemiology and End Results (SEER) program of the National Cancer Institute, the annual incidence rate of GEP-NETs is

Correspondence to: Professor Ping Chen, Department of Gastrointestinal Surgery, Clinical Medical College of Yangzhou University, Subei People's Hospital of Jiangsu, 98 Nantong West Road, Yangzhou, Jiangsu 225001, P.R. China

E-mail: chen86ky@126.com

Dr Liang Zong, Department of Gastrointestinal Surgery, Graduate School of Medicine, University of Tokyo, 7-3-1 Hongo, Bunkyo-ku, Tokyo 113-0033, Japan

E-mail: 250537471@qq.com

Key words: gastroenteropancreatic neuroendocrine tumors, gastrointestinal stromal tumors, stomach
3.65 cases per 100,000 individuals (4). Gastrointestinal stromal tumors (GISTs) are the most common type of mesenchymal tumor of the gastrointestinal system, and are derived from the interstitial Cajal cells of the gastrointestinal tract. GISTs are generally characterized by gain-of-function mutations in the KIT gene (5), and less often by PDGFRA or BRAF gene mutations (6-8). The annual incidence of GISTs ranges between 6.8 and 19.7 individuals per million across numerous countries, including the United States (9-11). GEP-NETs and GISTs are malignant or potentially malignant tumors, and are considered to have their own specific molecular biological behavior. As the majority of patients exhibit nonspecific symptoms, the identification of GEP-NETs and GISTs is often incidental. At present, diagnostic techniques include endoscopy, computed tomography (CT) and endoscopic ultrasonography (EUS) (12) and surgical resection remains the standard treatment for GEP-NETs and GISTs. The present study reports a compound case of GEP-NETs and GISTs that simultaneously occurred in the stomach.

\section{Case report}

A 56-year-old female was admitted to the Subei People's Hospital of Jiangsu (Yangzhou, Jiangsu, China) due to epigastric discomfort that had persisted for 4 weeks. Pre-operative blood examinations, including tumor marker analysis, were normal. Gastrointestinal endoscopy identified two fusiform masses of $1.8 \times 1.8 \mathrm{~cm}$ and $1.6 \times 1.6 \mathrm{~cm}$ located at the gastric body and gastric fundus, respectively (Fig. 1A and B). Meanwhile, EUS found that the two masses with low homogenous echogenicity each originated from the gastric muscular layer (Fig. 2A and B). Additionally, abdominal contrast-enhanced CT confirmed the presence of two well-marginated oval-shaped masses located at the gastric fundus and the lesser curvature of the cardia, respectively (Fig. 3). A pre-operative diagnosis of GISTs was formed.

At laparotomy, the tumor located at the lesser curvature of the cardia was found and measured $3 \times 3 \times 2.5 \mathrm{~cm}$ in size. The second tumor was found in the posterior wall of the gastric body and measured $\sim 1 \times 1 \times 0.5 \mathrm{~cm}$ in size. A proximal gastrectomy and Roux-en-Y reconstruction were performed.

Microscopically, the first tumor was found to be composed of spindle cells, a number of which showed high mitotic activity (Fig.4). Immunohistochemical staining showed that the tumor was positive for (CD)117, CD34, discovered on GIST-1 


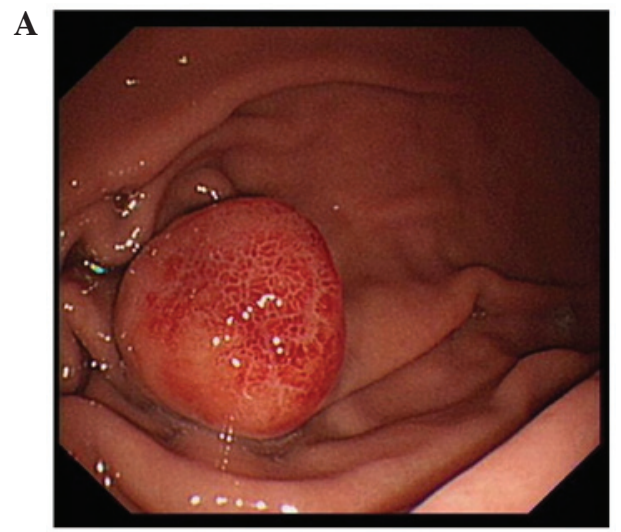

B

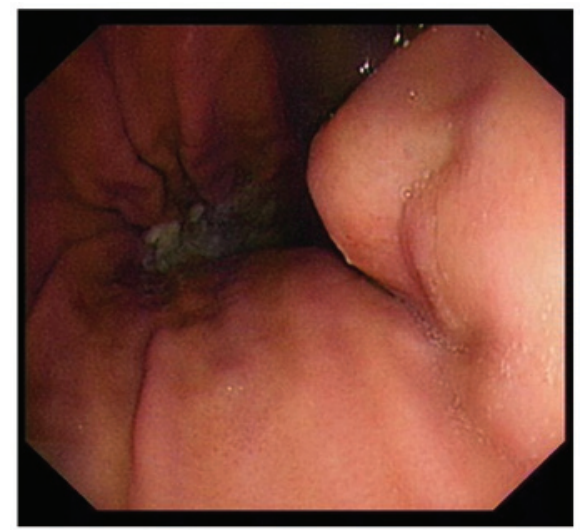

Figure 1. Pre-operative gastrointestinal endoscopy revealing a mass at the (A) gastric body and (B) the gastric fundus.

A

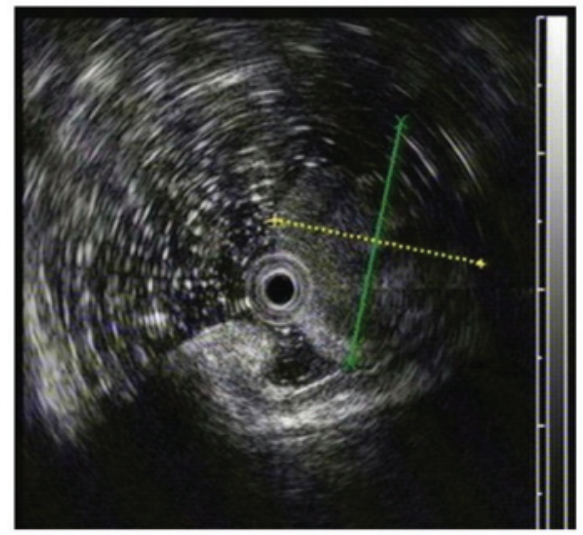

B

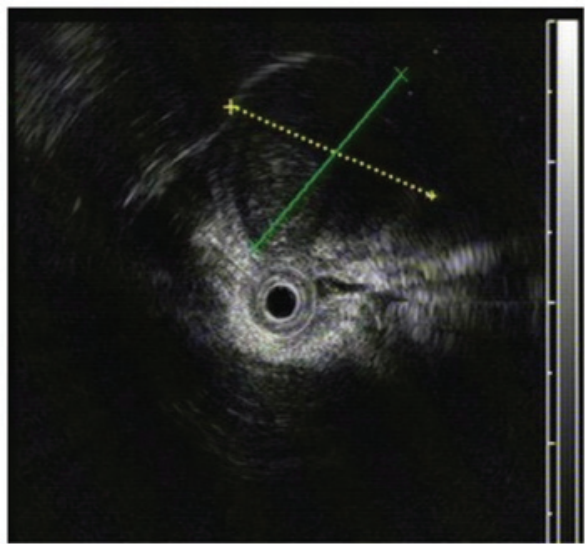

Figure 2. Pre-operative endoscopic ultrasonography shows tumors with low homogenous echogenicity originating in the (A) gastric body and (B) the gastric fundus.

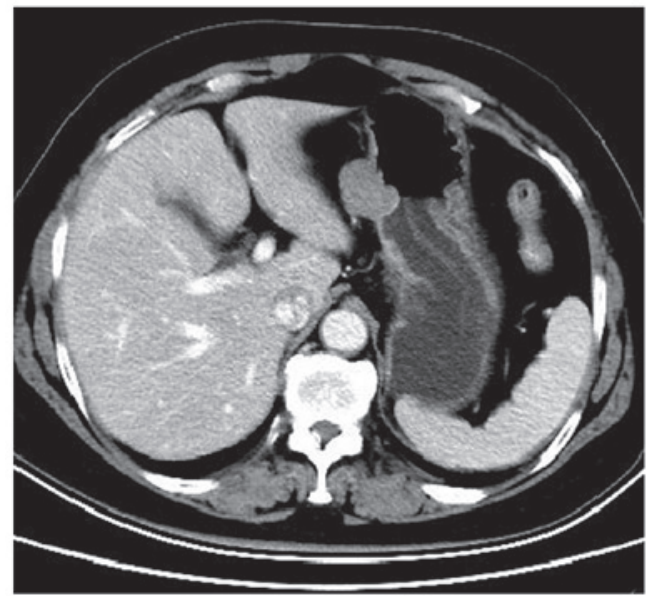

Figure 3. Pre-operative abdominal computed tomography demonstrating a single $3.1 \times 2.2-\mathrm{cm}$ mass located close to the gastric fundus and the upper lesser curvature of the stomach.

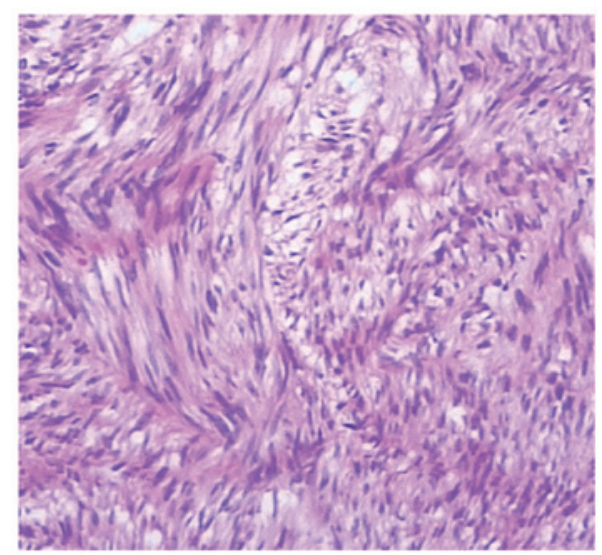

Figure 4. Hematoxylin and eosin-stained post-operative section. Swirling bundles of spindle cells with focally palisading areas within the fibrotic stroma were identified, but mitotic figures were rare (magnification, x100).

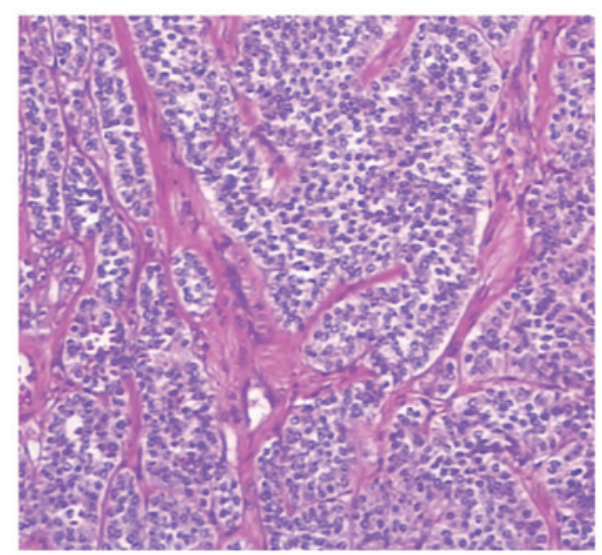

Figure 5.Hematoxylin and eosin-stained post-operative section. Neuroendocrine tumor cels were arranged in nests, with fibrous separation (magnification, x100).

and smooth muscle actin (SMA), but negative for desmin and S-100, suggesting a GIST origin.

Unexpectedly, the cells of the second tumor were organized in nests with fibrous separation (Fig. 5). The tumor cells 
exhibited a mitotic index of $<2 / 10$ high-power fields. Immunohistochemical results showed that the tumor was strongly positive for chromogranin $\mathrm{A}(\mathrm{CgA} ;+++)$, synaptophysin (Syn; $+++)$ and CD56 (+++), with a Ki-67 index of $<2 \%$. All the results suggested that this tumor was of GEP-NET origin.

Following the surgery, there were no post-operative complications. The patient was discharged from hospital after 10 days. After 17 months of follow-up examinations using CT and ultrasonography, the patient showed no symptoms or signs of recurrence. The present study was approved by the Subei People's Hospital of Jiangsu Ethical Committee and written informed consent was obtained from the patient.

\section{Discussion}

GEP-NETs originate from various neuroendocrine tissues of the gastroenteropancreatic system and form the largest subgroup of NETs (13). GEP-NETs usually and equally distribute in the foregut (stomach or duodenal), midgut (jejunal, ileal, appendix or proximal colon) and hindgut (distal colon or rectum) (14). Although they are rare neoplastic diseases in general, the incidence has greatly increased in the last decade (15).

In 2000, the World Health Organization classification system for NETs was published (16). Based on this system, proliferation index (Ki-67, MIB-1), angioinvasion and mitoses are regarded as the most important factors to determine the malignancy of NETs. NETs can be classified into the well-differentiated $(<2 \mathrm{~cm}$ in size, $<2 \% \mathrm{Ki}-67$ index), moderately-differentiated $(>2 \mathrm{~cm}$ in size, $>2 \% \mathrm{Ki}-67$ index, or angioinvasive) and poorly-differentiated (>20\% Ki-67 index) subtypes (17). In addition, the European Neuroendocrine Tumor Society proposed another grading system (G1, G2 and G3) for the classification of NETs (18). In general, G1 and G2 NETs refer to a well-differentiated subtype, displaying diffuse and intense expression of CgA and Syn. G3 NET indicates a poorly-differentiated subtype with high mitotic counts/Ki-67 index (>20\%), displaying slight staining of $\mathrm{CgA}$ and intense staining of Syn (19).

GISTs are also rare tumors, but are the most common mesenchymal tumor in the gastrointestinal tract (20). GISTs clinically present with characteristic GI bleeding, weight loss, abdominal pain, anemia and/or a palpable mass (21). The highest incidence of GISTs occurs in the stomach (52-60\%), followed by the small intestine $(20-30 \%)$ and colorectum (10\%). Approximately 95\% of GISTs are positive for c-Kit/CD117, 40-50\% for CD34, 20-30\% for SMA and $10 \%$ for S100 $(22,23)$. The National Institutes of Health and National Comprehensive Cancer Network systems were established to predict GIST behavior using risk assessment (very low risk, low risk, intermediate risk and high risk) (24).

To the best of our knowledge, the present study is the first to demonstrate a compound case of GEP-NETs and GISTs in the stomach. Surgical resection remains the first choice of treatment, however, due to the malignant potency of the two tumor types, long-term follow-up is greatly advised.

\section{References}

1. Jiao Y, Shi C, Edil BH, et al: DAXX/ATRX, MEN1, and mTOR pathway genes are frequently altered in pancreatic neuroendocrine tumors. Science 331: 1199-1203, 2011.
2. Modlin IM, Lye KD and Kidd M: A 5-decade analysis of 13,715 carcinoid tumors. Cancer 97: 934-959, 2003.

3. Modlin IM, Oberg K, Chung DC, Jensen RT, de Herder WW, Thakker RV, Caplin M, Delle Fave G, Kaltsas GA, Krenning EP, et al: Gastroenteropancreatic neuroendocrine tumours. Lancet Oncol 9: 61-72, 2008.

4. Lawrence B, Gustafsson BI, Chan A, Svejda B, Kidd M and Modlin IM: The epidemiology of gastroenteropancreatic neuroendocrine tumors. Endocrinol Metab Clin North Am 40: 1-18, 2011.

5. Hirota S, Isozaki K, Moriyama Y, et al: Gain-of-function mutations of c-kit in human gastrointestinal stromal tumors. Science 279: 577-580, 1998.

6. Heinrich MC, Corless CL, Duensing A, et al: PDGFRA activating mutations in gastrointestinal stromal tumors. Science 299: 708-710, 2003.

7. Hirota S, Ohashi A, Nishida T, et al: Gain-of-function mutations of platelet-derived growth factor receptor alpha gene in gastrointestinal stromal tumors. Gastroenterology 125: 660-667, 2003.

8. Daniels M, Lurkin I, Pauli R, et al: Spectrum of KIT/PDGFRA/ BRAF mutations and Phosphatidylinositol-3-Kinase pathway gene alterations in gastrointestinal stromal tumors (GIST). Cancer Lett 312: 43-54, 2011.

9. Reddy P, Boci K and Charbonneau C: The epidemiologic, health-related quality of life, and economic burden of gastrointestinal stromal tumours. J Clin Pharm Ther 32: 557-565, 2007.

10. Miettinen M and Lasota J: Histopathology of gastrointestinal stromal tumor. J Surg Oncol 104: 865-873, 2011.

11. Robinson TL, Sircar K, Hewlett BR, Chorneyko K, Riddell RH and Huizinga JD: Gastrointestinal stromal tumors may originate from a subset of CD34-positive interstitial cells of Cajal. Am J Pathol 156: 1157-1163, 2000.

12. Ramage JK, Ahmed A, Ardill J, et al; UK and Ireland Neuroendocrine Tumour Society: Guidelines for the management of gastroenteropancreatic neuroendocrine (including carcinoid) tumours (NETs). Gut 61: 6-32, 2012.

13. Oberg K: Somatostatin-receptor mediated diagnosis and treatment in gastrointestinal neuroendocrine tumours (GEP-NET's). Rocz Akad Med Bialymst 50: 62-68, 2005.

14. Klöppel G and Anlauf M: Epidemiology, tumour biology and histopathological classification of neuroendocrine tumours of the gastrointestinal tract. Best Pract Res Clin Gastroenterol 19: 507-517, 2005.

15. Schimmack S, Svejda B, Lawrence B, Kidd M and Modlin IM: The diversity and commonalities of gastroenteropancreatic neuroendocrine tumors. Langenbecks Arch Surg 396: 273-298, 2011.

16. Hamilton SR and Aaltonen LA (eds): World Health Organization Classification of Tumors. Pathology and Genetics of Tumours of the Digestive System. IARC Press, Lyon, 2000.

17. Modlin IM, Moss SF, Oberg K, Padbury R, Hicks RJ, Gustafsson BI, Wright NA and Kidd M: Gastrointestinal neuroendocrine (carcinoid) tumours: Current diagnosis and management. Med J Aust 193: 46-52, 2010.

18. Klöppel G1, Couvelard A, Perren A, et al; Mallorca Consensus Conference participants; European Neuroendocrine Tumor Society: ENETS Consensus Guidelines for the Standards of Care in Neuroendocrine Tumors: Towards a standardized approach to the diagnosis of gastroenteropancreatic neuroendocrine tumors and their prognostic stratification. Neuroendocrinology 90: 162-166, 2009.

19. Oberg K and Castellano D: Current knowledge on diagnosis and staging of neuroendocrine tumors. Cancer Metastasis Rev 30 (Suppl 1): 3-7, 2011.

20. George S and Desai J: Management of gastrointestinal stromal tumors in the era of tyrosine kinase inhibitors. Curr Treat Options Oncol 3: 489-496, 2002.

21. Rabin I, Chikman B, Lavy R, Sandbank J, Maklakovsky M, Gold-Deutch R, Halpren Z, Wassermann I and Halevy A: Gastrointestinal stromal tumors: A 19 year experience. Isr Med Assoc J 11: 98-102, 2009.

22. Corless CL, Fletcher JA and Heinrich MC: Biology of gastrointestinal stromal tumors. J Clin Oncol 22: 3813-3825, 2004

23. Kang YN, Jung HR and Hwang I: Clinicopathological and immunohistochemical features of gastointestinal stromal tumors. Cancer Res Treat 42: 135-143, 2010.

24. National Comprehensive Cancer Network (NCCN): NCCN Clinical Practice Guidelines in Oncology. Soft Tissue Sarcoma. v.2.2009 [Internet]. National Comprehensive Cancer Network, Fort Washington, PA, 2009. 